\title{
Parallelizable Authentication Trees
}

\author{
W. Eric Hall and Charanjit S. Jutla \\ IBM T.J. Watson Research Center, Yorktown Heights, NY 10598-704
}

\begin{abstract}
We define a new authentication tree in the symmetric key setting, which has the same computational time, storage and security parameters as the well known Merkle authentication tree, but which unlike the latter, allows for all the cryptographic operations required for an update to be performed in parallel. As in Merkle authentication trees, the cryptographic operations required for verification can also be parallelized. In particular, we show a provably secure scheme for incremental MAC with partial authentication secure against substitution and replay attacks, which on total data of size $2^{n}$ blocks, and given $n$ cryptographic engines, can compute incremental MACs and perform individual block authentication with a critical path of only one cryptographic operation
\end{abstract}

\section{Introduction}

We design a novel incremental MAC (message authentication code) with partial authentication secure against substitution and replay attacks. Before we give detailed definitions, to motivate the definitions and as an application, consider the following problem of checking correctness of memory [4].

In this application, a tamper proof processor uses an insecure storage device (e.g. RAM), open to attack from an adversary who can read and modify the RAM. However, the tamper proof processor may also have a small amount of internal memory which can store a MAC of the whole unprotected RAM. The tamper proof processor is required to generate incremental MACs and authenticate individual blocks of RAM (without computing the MAC on the whole RAM). Of course, these computations should not substantially deteriorate the performance of the overall system. A similar situation arises in NAS (network attached storage) systems.

Clearly, there are two extreme (though impractical) solutions to this problem. One is to store the whole RAM (say $2^{n}$ blocks) inside the tamper proof device. The other is to have $2^{n}$ cryptographic engines (e.g. AES or SHA-1) inside the tamper proof device which can compute/authenticate a MAC of the whole unprotected memory using a parallel MAC scheme like XOR-MAC ([1], also see section 6), with a critical path of one cryptographic operation. We stress here that although XOR-MAC can compute incremental MACs with only one engine, to verify an individual block of RAM, it must compute the MAC on the whole RAM (i.e. XOR-MAC is not incremental with respect to verification). The ability to verify only a single block (without having to compute the whole MAC) is a crucial requirement of our problem.

One could try a memory/processor tradeoff, by dividing the unprotected memory into super-blocks (say of size $2^{m}$ blocks each), and storing an 
XOR-MAC (one block) of each super-block inside the tamper proof device, and computing/authenticating an XOR-MAC in parallel using $2^{m}$ cryptographic engines. Now the tamper proof memory required to store the MACs has been reduced to $2^{n-m}$ blocks 1 . Note however, that the number of engines plus the tamper proof memory required remains at least $2^{n / 2}$.

The main contribution of this paper is a provably secure scheme for this problem, which with only $n$ cryptographic engines, and 1 block of tamper proof memory, can compute incremental MACs (and do individual block authentication) with a critical path of one cryptographic operation. The only overhead is an increase in size of the unprotected memory by a factor of two.

Before we describe our scheme, lets describe the other existing solution to this problem. This solution of [4] uses Merkle authentication trees [13]. However, Merkle trees are not fully parallelizable (i.e. although a Merkle tree based solution would only require $n$ cryptographic engines, and 1 block of tamper proof memory, the critical path of an incremental MAC computation would be $n$ cryptographic operations). Not surprisingly though, as we will soon see, ours is also a tree based solution. In section 7 we contrast our scheme with Merkle Trees in more practical settings, and also elaborate on other systems issues.

Main Result. As for XOR-MAC, for every finite secure PRF (pseudorandom function) family $F$, we construct an incremental $M A C$ with partial authentication secure against substitution and replay attacks. The key difference from XOR$\mathrm{MAC}$ is that our scheme does efficient parallel partial authentication. To achieve our goal, the scheme we describe generates auxiliary data which can be stored in unprotected memory. In other words, when provided with correct auxiliary data a single block can be authenticated, whereas no adversary can get a wrong block authenticated even with auxiliary data of its choice.

Surprisingly, the MAC is just a nonce value chosen independently of the data to be authenticated! It is the auxiliary data which provides complete authentication, and in a way we are trying to make the naive solution of "storing MACs outside in unprotected memory" work (see footnote). Informally, the scheme works as follows. Each pair of data blocks is locally MACed along with a new nonce value (chosen randomly or deterministically), and each pair of these nonce values are locally MACed along with yet another new nonce value at a higher level, and so on, till we reach the root of this tree. The new nonce value at the root is the MAC of the scheme. The local MACs are all stored outside in unprotected memory as auxiliary data. We stress that we are not locally MACing two child nodes with the value at the parent as key, but rather MACing all three values together using a global secret key. The former would lead to an insecure solution, because the parent value (if used as the MAC key) would be available to the adversary. The latter can be seen as a tweakable MAC (cf. tweakable block ciphers [1], 10]), i.e. we use the parent value as a tweak.

\footnotetext{
${ }^{1}$ Note that storing MACs outside in unprotected memory provides only integrity, and not protection from replay attacks.
} 
Note that all the local computations can be done in parallel once the nonces have been chosen. Efficient incrementality follows as an update in a data block only requires updates on the path from this leaf to the root. Efficient parallel partial authentication, i.e. authentication of data at a leaf, follows as it requires checking the local MACs on the path from this leaf to the root.

The only thing that remains to be seen is that we do not reuse nonce values, and that the adversary cannot move around the auxiliary data including replaying old auxiliary data, which is the crux of the proof of security.

We finally describe how an XOR-MAC like scheme PMAC [3] (which uses XOR universal hash function families [8], [5]) can be extended to provide efficient partial authentication.

The rest of the paper is organized as follows. In section 2, we define a novel notion of incremental MAC with partial authentication, and give its definition of secutiy. In section 3, we define our scheme PAT, while the proofs of security are given in section 4 . Further optimizations are given in Section 5 . In section 6 , we describe PMAC Trees, an extension of PMAC which allows partial authentication. In section 7, we discuss various practical issues like cache-based and pipelined systems, and contrast our scheme with both Merkle Trees and PMAC Trees.

\section{Definitions}

As is often conveniently done, for a function $F$ with many parameters, if the first parameter is a key then $F_{x}$ will denote the function with the first parameter $x$. The symbol || will denote concatenation of strings. For a message $M$ consisting of $m$ blocks $M_{1}, \ldots, M_{m}, M\langle i, a\rangle$ will denote the modified message with the $i$ th block $M_{i}$ replaced by $a$.

We now define simple Message Authentication Codes(MAC)and their security.

Definition 2.1. A simple MAC scheme consists of a function $F$ which takes a secret key $x$ of $k$ bits, a plaintext $M$ of $m$ bits and produces a value $\tau$ of $h$ bits.

Security of the simple MAC scheme is defined using the following experiment. An oracle adversary $A$ is given access to the oracle $F_{x}(\cdot)$. After $A$ queries $F_{x}(\cdot)$ on plaintexts $M_{1}, M_{2}, \ldots, M_{q}$ (adaptively), it outputs a pair $M^{\prime}, \tau^{\prime}$, where $M^{\prime}$ is not one of the queried plaintexts. The adversary's success probability is given by

$$
\operatorname{Pr}_{x}\left[\mathrm{~F}_{x}\left(M^{\prime}\right)=\tau^{\prime}\right]
$$

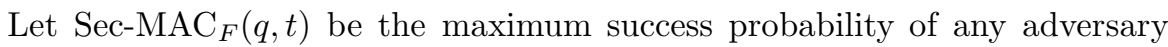
running in time at most $t$, and making at most $q$ queries.

The above security is known as security under the impersonation attack. There is another notion of security called the substitution attack. In this model, the adversary $A$ works as follows. After $A$ queries $F_{x}(\cdot)$ on plaintexts $M_{1}, M_{2}, \ldots, M_{q}$ (adaptively), and the algorithm returns $\tau_{1}, \tau_{2}, \ldots, \tau_{q}$, the adversary then outputs an $M^{\prime}$. The adversary's success probability is given by

$$
\operatorname{Pr}\left[\exists j 1 \leq j \leq q: \mathrm{F}_{x}\left(M^{\prime}\right)=\tau_{j} \wedge M^{\prime} \neq M_{j}\right]
$$


Finally, there is a third notion of security called the replay attack. In this model, the adversary works as follows. After $A$ queries $F_{x}(\cdot)$ on plaintexts $M_{1}, M_{2}, \ldots, M_{q}$ (adaptively), and the algorithm returns $\tau_{1}, \tau_{2}, \ldots, \tau_{q}$, the adversary then outputs a pair $M^{\prime}, \tau^{\prime}$, such that $M^{\prime}$ is just required to be different from $M_{q}$. The adversary's success probability is given by

$$
\operatorname{Pr}\left[\mathrm{F}_{x}\left(M^{\prime}\right)=\tau^{\prime}\right]
$$

Of course, there is an adversary which always manages a replay attack. However, the notion can be strengthened by requiring that $\tau^{\prime}$ must agree with some portion of $\tau_{q}$. As we will see, this is an important notion for incremental MACs.

To the best of our knowledge the next two definitions are novel.

Definition 2.2. An incremental $M A C$ with partial authentication and with auxiliary data (IMACAUX) consists of the following:

- MAC-AUX: MAC-AUX is a probabilistic function with arguments a key $x$ of $k$ bits, and a plaintext $M$ of size at most $2^{n}$ blocks, each block being of size $m$ bits, and which produces a tuple $\langle\sigma, \tau\rangle$, where $\sigma$ can be an arbitrarily long string, and $\tau$ is of size $h$ bits. The string $\sigma$ will be called the auxiliary data and $\tau$ will be called the authentication tag.

We will write $\left[\mathrm{MAC}-\mathrm{AUX}_{x}(M)\right]$ for all tuples $\langle\sigma, \tau\rangle$ which have non-zero

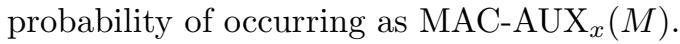

- Verify: Verify is a boolean function which takes a key $x$ of $k$ bits, an index $i \in\left[0 . .2^{n}-1\right]$, an $m$ bit block $a$, auxiliary data $\sigma$ and $\operatorname{tag} \tau$, with the following property: $\operatorname{Verify}_{x}(i, a, \sigma, \tau)$ must return 1 if there exists an $M$, such that the $i$ th block of $M$ is $a$, and $\langle\sigma, \tau\rangle$ is in [MAC-AUX $\left.\operatorname{AM}_{x}(M)\right]$.

- INC-MAC-AUX (update): INC-MAC-AUX is a probabilistic function which takes (apart from the key $x$ ) an index $i$, a block of plaintext $a$, auxiliary data $\sigma$, and $\operatorname{tag} \tau$, and produces either a tuple $\left\langle\sigma^{\prime}, \tau^{\prime}>\right.$ or $\perp$. If there exists an $M$ such that $\langle\sigma, \tau\rangle$ is in $\left[\operatorname{MAC}-\operatorname{AUX}_{x}(M)\right]$ then it must return

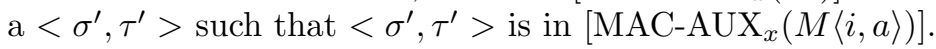

We note that one way to implement the above is to embed $M$ in the auxiliary data $\sigma$. We have said nothing about the security of IMACAUX, which we address next.

Definition 2.3 (Security under substitution and replay attacks). The security of an IMACAUX scheme 〈 MAC-AUX, Verify, INC-MAC-AUX〉 is defined using the following experiment. A three oracle adversary $A$ is given access to the oracles $\operatorname{MAC}_{-A_{X}}(\cdot)$, Verify ${ }_{x}(\cdot, \cdot, \cdot, \cdot)$, and INC-MAC-AUX ${ }_{x}(\cdot, \cdot, \cdot, \cdot)$. The adversary first requests an initial $\mathrm{MAC}^{-\mathrm{AUX}_{x}}$ to be computed on an initial plaintext $M^{0}$ of $2^{n}$ blocks. Let $\mathrm{MAC}_{-\mathrm{AUX}_{x}}\left(M^{0}\right)$ return $<\sigma_{0}, \tau_{0}>$.

Subsequently, the adversary requests a sequence of $q$ adaptive update operations, each specifying a block number and a block of replacement text, along with auxiliary data of its choice. However, $\tau$ supplied on each request must be same as that produced in the previous request. 
More precisely, for each $j, 0<j \leq q$, let $i_{j}$ be the block number for the $j$ th incremental update, with text $a_{j}$. Let $I_{j}=\operatorname{INC-MAC-AUX}\left(i_{j}, a_{j}, \sigma_{j-1}^{\prime}, \tau_{j-1}\right)$, where $i_{j}, a_{j}$, and $\sigma_{j-1}^{\prime}$ are adaptively chosen by the adversary. As required in definition 2.2, the return value $I_{j}$ is either $\perp$ or $\left\langle\sigma_{j}, \tau_{j}\right\rangle$. If the return value is $\perp$, then we let $M^{j}=M^{j-1}$ and $\tau_{j}=\tau_{j-1}$. If the return value is $\left\langle\sigma_{j}, \tau_{j}>\right.$ then we let $M^{j}=M^{j-1}\left\langle i_{j}, a_{j}\right\rangle$. In the latter case we also say the update was valid.

Finally, the adversary requests a verification on a block at index $i$, with text $a$, such that $a$ is different from $M_{i}^{q}$. The adversary's success probability is given by

$$
\operatorname{Pr}_{x}\left[\operatorname{Verify}_{x}\left(i, a, \sigma^{\prime}, \tau_{q}\right)=1\right]
$$

Again, $\sigma^{\prime}$ is adaptively chosen by the adversary. However, $\tau_{q}$ remains the same as produced by the last valid update. We stress that $a$ is only required to be different from the last plaintext block at index $i$, and the adversary is allowed to choose an $a$ (along with a related $\sigma^{\prime}$ ) which may have occurred at an earlier point of time at index $i$.

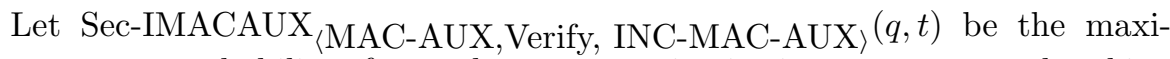
mum success probability of any adversary running in time at most $t$, and making at most $q$ INC-MAC-AUX requests.

\section{Parallelizable Authentication Tree}

We now describe an IMACAUX scheme called PAT with a description of each of its component functions, i.e. MAC-AUX, Verify and INC-MAC-AUX. The functions will employ a simple MAC scheme $F$ (see definition 2.1) with the same secret key $x$ as chosen for PAT. We will describe the various size parameters later (before Theorem 1). All $F$ computations (which will be the only cryptographic operations) in the computation of these functions can be done in parallel.

MAC-AUX. Given a $2^{n}$ block plaintext $M$, we now describe a valid MAC-AUX on it, i.e. all pairs $\langle\sigma, \tau\rangle$ which are in $\left[\operatorname{MAC}_{-} \operatorname{AUX}_{x}(M)\right]$. $\operatorname{MAC}_{-A_{X}}(M)$ will be an $r$-ary labeled tree (see Figure 1 ). For simplicity, we only consider $\mathrm{r}=2$. The tree will be balanced and will have $2^{n}$ leaves. For a non-leaf node $u$, left $(u)$ will denote its left child and $\operatorname{right}(u)$ will denote its right child.

- Leaf nodes $u$ will have as label a data value $\operatorname{data}(u)$ which will be the corresponding block from $M$.

- the labels at each non-leaf node $u$ will be a nonce value $V(u)$, and a local mac value $C(u)$ such that $C(u)=\mathrm{F}_{x}(V(l e f t(u))\|V(u)\| V($ right $(u)))$, where $\|$ is the concatenation operator (see Fig 1 ).

The auxiliary data $\sigma$ is just the whole labeled tree except the root $V$ label. The authentication $\operatorname{tag} \tau$ is just the $V$ label of the root node. Thus, $\langle\sigma, \tau\rangle$ is the whole labeled tree. Any such $\langle\sigma, \tau\rangle$, which satisfies the local $F$ constraints, is in $[\mathrm{MAC}-\mathrm{AUX}(M)]$.

Verify. Let the input to the function be $(i, a, \sigma, \tau)$. 


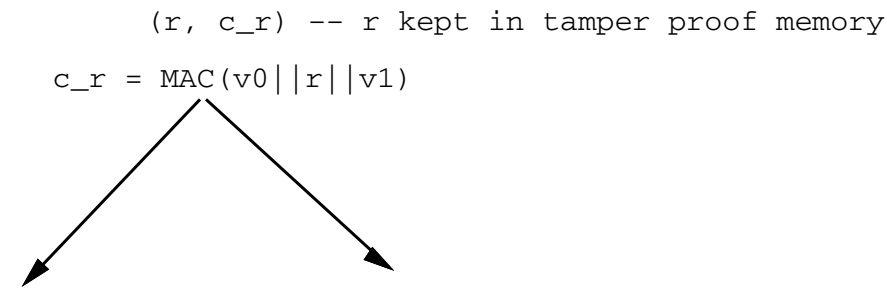

$(\mathrm{v} 0, \mathrm{c} 0)$

$\mathrm{C} 0=\mathrm{MAC}(\mathrm{v} 00|| \mathrm{v} 0|| \mathrm{v} 01)$

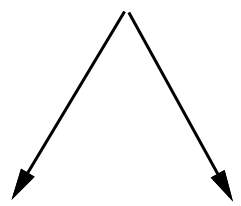

$(\mathrm{v} 00, \mathrm{c} 00)$

$$
(\mathrm{v} 01, \mathrm{c} 01)
$$

$(\mathrm{v} 1, \mathrm{c} 1)$

$\mathrm{c} 1=\mathrm{MAC}(\mathrm{v} 10|| \mathrm{v} 1|| \mathrm{v} 11)$

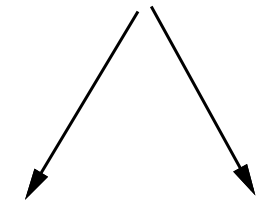

$(\mathrm{v} 10, \mathrm{c} 10) \quad(\mathrm{v} 11, \mathrm{c} 11)$
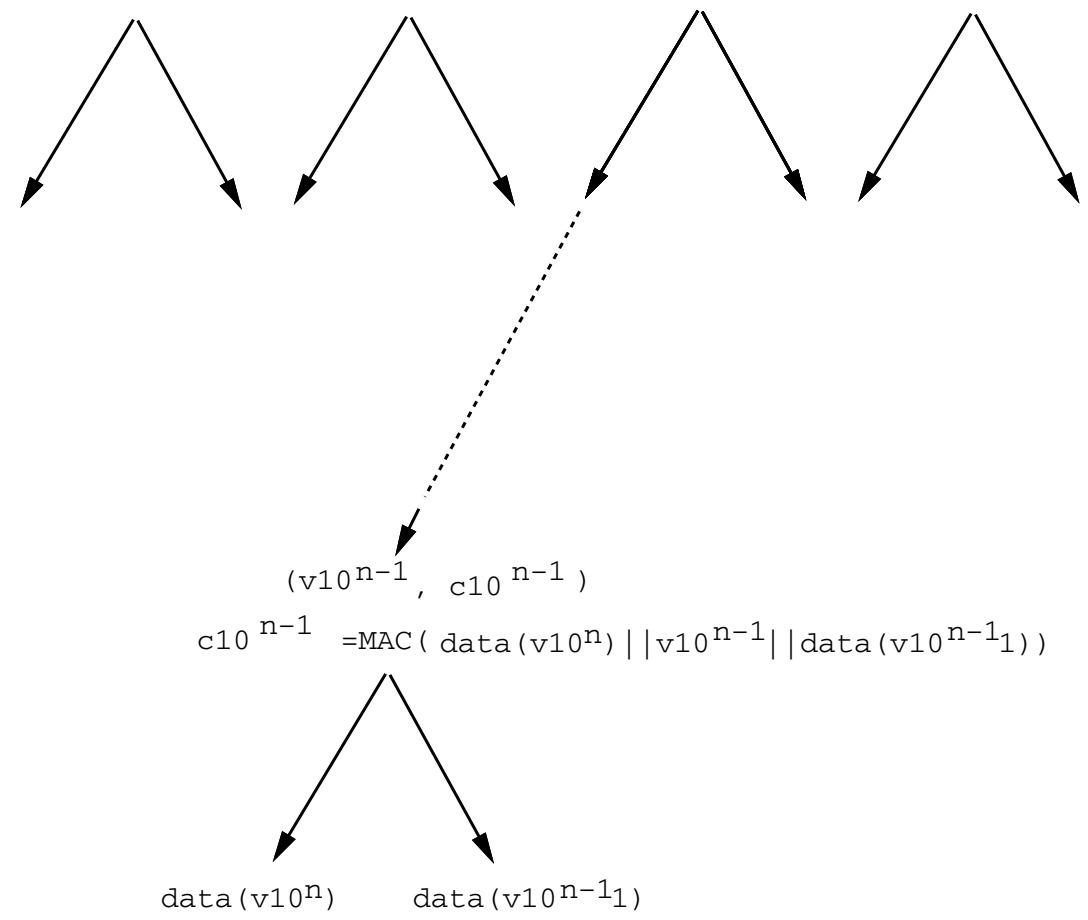

Fig. 1. Parallelizable Authentication Tree (PAT)

Since the boolean function Verify takes the leaf node index as an argument (i.e. $i$ ), it just checks that the nonce values are consistent with $C$ along the path from the root to this leaf. We will give an algorithmic description of this function. 
More precisely, let the path from the root to the specified leaf $z$ (the $i$ th leaf) be $u_{0}=r, u_{1}, \ldots, u_{n-1}, u_{n}=z$. Recall that $V\left(u_{0}\right)$ is $\tau$.

If for all levels $y=0$ to $n-2, C\left(u_{y}\right)$ equals

$\mathrm{F}_{x}\left(V\left(\operatorname{left}\left(u_{y}\right)\right)\left\|V\left(u_{y}\right)\right\| V\left(\operatorname{right}\left(u_{y}\right)\right)\right)$, and

if $u_{n}$ is the left child of $u_{n-1}$ then $C\left(u_{n-1}\right)$ equals

$\mathrm{F}_{x}\left(a\left\|V\left(u_{n-1}\right)\right\| \operatorname{data}\left(\operatorname{right}\left(u_{n-1}\right)\right)\right)$, and

if $u_{n}$ is the right child of $u_{n-1}$ then $C\left(u_{n-1}\right)$ equals

$\mathrm{F}_{x}\left(\operatorname{data}\left(\operatorname{left}\left(u_{n-1}\right)\right)\left\|V\left(u_{n-1}\right)\right\| a\right)$

then return 1 , else return 0 .

Note that the nonce values $\mathrm{V}$ and the data values in the above verification are given as part of $\sigma$.

INC-MAC-AUX (update). Let the input to the function be $(i, a, \sigma, \tau)$. We will give an algorithmic description of this function.

Since the tree has $2^{n}$ leaves, the root can be considered to be at the 0th level, whereas the leaves are at the $n$th level. A path from root $r$ to leaf $z$ (the $i$ th leaf) can then be written as $u_{0}=r, u_{1}, \ldots, u_{n-1}, u_{n}=z$. This will be called the update path. Let $v_{1}, \ldots v_{n}$ be the sibling path, i.e. for each $y \in[1 . . n], v_{y}$ is the sibling of $u_{y}$. The update algorithm first checks that the nonce values $V$ provided as part of $\sigma$ of the update and sibling path nodes are correct. More precisely,

Step 1 : If for all $y=0$ to $n-2$,

$$
C\left(u_{y}\right) \text { equals } \mathrm{F}_{x}\left(V\left(\operatorname{left}\left(u_{y}\right)\right)\left\|V\left(u_{y}\right)\right\| V\left(\operatorname{right}\left(u_{y}\right)\right)\right) \text {, and }
$$

$C\left(u_{n-1}\right)$ equals $\mathrm{F}_{x}\left(\right.$ data $\left.\left(\operatorname{left}\left(u_{n-1}\right)\right)\left\|V\left(u_{n-1}\right)\right\| \operatorname{data}\left(\operatorname{right}\left(u_{n-1}\right)\right)\right)$, then perform the update below, else return $\perp$.

Step 2 : For $y=0$ to $n-1$, update $V\left(u_{y}\right)=\tau+n-y$.

For $y=0$ to $n-2$, update $C\left(u_{y}\right)=\mathrm{F}_{x}\left(V\left(\operatorname{left}\left(u_{y}\right)\right)\left\|V\left(u_{y}\right)\right\| V\left(\operatorname{right}\left(u_{y}\right)\right)\right)$.

Step 3 : At the leaf node $z=u_{n}$, update $\operatorname{data}\left(u_{n}\right)=a$, and update $C\left(u_{n-1}\right)=\mathrm{F}_{x}\left(\operatorname{data}\left(\operatorname{left}\left(u_{n-1}\right)\right)\left\|V\left(u_{n-1}\right)\right\| \operatorname{data}\left(\operatorname{right}\left(u_{n-1}\right)\right)\right)$.

The newly re-labeled tree is returned as $\sigma, \tau$. Note that $\tau$ is just the new $V\left(u_{0}\right)$, i.e. old $\tau$ plus $n$. Note that all the $F$ operations in steps 1 to 3 combined can be done in parallel. If these steps are indeed done in parallel in some implementation, there could be a possibility that Step 1 fails, in which case step 2 and 3 are abandoned, and the old $\sigma, \tau$ is reverted to. Since the chances of Step 1 failing are hopefully small, this does not incur much cost.

That finishes the description of INC-MAC-AUX.

\section{Security of Parallelizable Authentication Tree}

\subsection{Optimized Initialization of the Authentication Tree}

Since, in the definition of security which described the adversarial model (see definition 2.3), the adversary only makes one initial call to MAC-AUX, our scheme (i.e. MAC-AUX) will return a $\sigma_{0}$, with all $V$ values set to zero, and similarly the $\tau_{0}$ set to zero. This can be seen as initializing the data structure. 
Moreover, with this simple initialization, all the internal node MAC values $C$ are the same, and hence need not be computed multiple times. Further still, we will assume that the data values are also initialized to zero, in which case the MAC values at the leaves will also be same.

This does not change the adversarial model, since if the adversary requested a different initial plaintext $M^{0}$, our algorithm could return the requested data structure by simulating several updates.

For example, before the first update request, as mentioned all nonce values are zero. After the first update request, the nonce value of the node closest to the leaf, i.e. $u_{n-1}$ will be 1 , and the nonce value of the node closest to the root, i.e. $u_{1}$ will be $n-1$, and the nonce value of the root will just be $n$.

As we will see, this assures that in each incremental request, the nonce values are chosen afresh, i.e. are never repeated.

Let $d$ be the number of bits in the nonce labels $V$ above.

Let $h$ be the number of bits in the $\mathrm{C}$ label.

Let $m$ be the number of bits in each block of data stored at a leaf.

Let $k$ be the number of bits in the key.

Let $2^{n}$ be the number of leaves in the balanced binary tree.

Let $F$ be a function $F:\{0,1\}^{k} \times\{0,1\}^{\max \{d, m\}+d+1} \rightarrow\{0,1\}^{h}$

The above three algorithms together describe an IMACAUX scheme and will be called $P A T^{F}(k, m, n, h, d)$ (parallelizable authentication tree) when using $F$ as its local MAC algorithm.

In the following theorem, Sec-MAC $F$ refers to the security under the impersonation attack in definition 2.1 .

Theorem 1: For any positive integers $k, m, n, h, d, q, t$ and any function $F:\{0,1\}^{k} \times\{0,1\}^{\max \{d, m\}+d+1} \rightarrow\{0,1\}^{h}$

$$
\operatorname{Sec}_{-M_{F}}(2 q n, t) \geq \operatorname{Sec}-\operatorname{IMACAUX}_{P A T^{F}(k, m, n, h, d)}(q, t)
$$

Proof: Let A be a three oracle adversary as in the experiment of Theorem 2.3. Let $\mathrm{B}$ be an oracle adversary which participates in the experiment of Definition 2.1 (simple MAC scheme), and is given access to the oracle $F_{x}(\cdot)$, with $x$ chosen uniformly at random.

Adversary B will simulate the three oracles for A, i.e. MAC-AUX, Verify, and INC-MAC-AUX of PAT using its own oracle $F_{x}(\cdot)$. B will then just imitate A. During the simulation, B will make several oracle calls to $F_{x}(\cdot)$. It will also maintain a List of pairs. Let List ${ }_{j}$ denote all pairs $(a, b)$ till the end of the $j$ th INC-MAC-AUX (update) query made by A, such that B during its simulation made a call to $F_{x}$ with input $a$ and $F_{x}(a)$ returned $b$. Ultimately, while simulating the final Verify query of A (or even during the INC-MAC-AUX queries' step 1, which is essentially a verify query), we show that for some $a$ and $b$ determined by $\mathrm{A}$, such that $a$ has not been a query to $F_{x}(\cdot)$, the verify query returns 1 iff $F_{x}(a)$ equals $b$. This claim proves the theorem.

We will follow notation from definition 2.3 for all the queries of adversary A. 
We say that a node $u$ was assigned a value by the algorithm PAT in the $j$ th update query if the $j$ th update is valid and this node is in the update path of the $j$ th query. Clearly, the root is assigned a value in each valid update query, and a leaf is assigned a value in an update query only if it was the leaf being updated and the update was valid. For each node $v$, let $\operatorname{last}(v, j)$ be the largest $j^{\prime} \leq j$ such that $v$ was assigned a value in the $j^{\prime}$ th query. If it was never assigned a value in an update query $(\leq j)$ then $\operatorname{last}(v, j)=0$. Let latest $(v, j)$ be the $V$ value assigned to $v$ by the algorithm INC-MAC-AUX (of PAT) in query $\operatorname{last}(v, j$ ); if $v$ is a leaf node then $\operatorname{latest}(v, j)$ is just the data assigned to that leaf in the $\operatorname{last}(v, j)$ query. The initial MAC-AUX query will be considered as the 0th query. Without loss of generality, assume that all updates retuen non- $\perp$ values (i.e. are valid updates), as updates which return $\perp$ do not cause any change in state. Fact 1(a) below follows from the optimized initialization of the authentication tree.

Fact 1: (a) For all $u$, latest $(u, 0)=0$.

(b) For $l \geq 1$, for all $u$ if $\operatorname{last}(u, l)=l$, then $\operatorname{latest}(u, l)=l * n-\operatorname{level}(u)$, where level $(u)$ is the distance of $u$ from the root.

(c) For all $u, j$ and $l$, if $\operatorname{last}(u, j) \leq l$, then $\operatorname{latest}(u, j)=\operatorname{latest}(u, l)$.

Claim 2: for any non-leaf nodes $u, v, u \neq v$, for all $t, t^{\prime}$, latest $(u, t) \neq$ latest $\left(v, t^{\prime}\right)$, or latest $(u, t)=\operatorname{latest}\left(v, t^{\prime}\right)=0$.

Proof: Suppose both values are non-zero. Then by fact 1(a) and 1(c), let $\operatorname{last}(u, t)=l \geq 1$, and $\operatorname{last}\left(v, t^{\prime}\right)=l^{\prime} \geq 1$. Then by Fact $1(\mathrm{~b})$, latest $(u, t)=$ $l * n-\operatorname{level}(u)$, and latest $\left(v, t^{\prime}\right)=l^{\prime} * n-\operatorname{level}(v)$. It follows that if these two values are same then $\operatorname{level}(u)=\operatorname{level}(v)$, and $l=l^{\prime}($ as $|\operatorname{level}(u)-\operatorname{level}(v)|<n)$. But that is impossible, as in the $l$ th update query only one node at each level gets a new $V$ value.

In the algorithm INC-MAX-AUX, each node in the update path and the sibling path is first verified. Define $S_{j}(1 \leq j \leq q)$ to be the union of nodes in the update and sibling path in the $j$ th update query. We will also call the final Verify query of adversary A, the $(q+1)$ th query. We define $S_{q+1}$ to be the collection of nodes in the path from the leaf being verified to the root.

Unless otherwise mentioned, whenever we refer to $V(u)$ for some node, we will mean the $V$ value supplied for node $u$ by adversary in the $j$ th query. The same will hold for $\operatorname{data}(u)$.

Claim 3: Either (a) for every $j, 1 \leq j \leq q+1$, for all nodes $u \in S_{j}, V(u)$ (or $\operatorname{data}(u))=$ latest $(u, j-1)$, or

(b) there exists a $j, 1 \leq j \leq q+1$, and a non-leaf node $u \in S_{j}$ such that $(V(\operatorname{left}(u))\|V(u)\| V(\operatorname{right}(u)))$ is not in $\operatorname{List}_{j-1}$ (i.e. is not equal to the first entry of any pair in List $\left._{j-1}\right)$.

Proof: Suppose (b) does not hold. Then we prove (a) by induction on $j$. 
Base case $(j=1)$. By Fact $1(\mathrm{a})$, for all $u$, latest $(u, j-1)=0$. Hence, the only entry in List $_{0}$ is the one corresponding to argument $(0\|0\| 0)$. Now, suppose (a) does not hold for some $u \in S_{j}$ (i.e. $\mathrm{V}(u) \neq 0$ ). If $u$ is a non-leaf node then (b) does not hold for that $u$ (with $j=1$ ). If $u$ is a leaf node then (b) does not hold for parent of $u$.

Suppose that the induction hypothesis holds for $j-1$.

We do a nested induction on the nodes of the update path. If $j<q+1$, consider a non-leaf node $u$ in $S_{j}$, but restricted to the update path. If $j=q+1$, then consider a non-leaf node $u$ in $S_{q+1}$. Suppose its supplied $V(u)$ is indeed same as latest $(u, j-1)$. We will show that for $v$ being either child of $u, V(v)$ (or $\operatorname{data}(v))$ supplied in the $j$ th query is indeed latest $(v, j-1)$.

Let $\operatorname{last}(u, j-1)$ be $l \leq j-1$. If $l=0$, then both its children $v$ have $V(v)=$ $0=\operatorname{latest}(v, j-1)$. Otherwise $u$ is in $S_{l}$ and the $l$ th update was valid. Now, since $\operatorname{last}(u, j-1)=l$, neither of $u$ 's children $v$ have $\operatorname{last}(v, j-1)>l$. In fact one of them, say $v 1$, has $\operatorname{last}(v 1, j-1)=l$, and the other, say $v 2$, has last $(v 2, j-1)<l$ (i.e. $v 2$ is in the sibling path in the $l$ th query). Moreover, $v 2$ is also in $S_{l}$. Thus, by outer induction hypothesis, $V$ value supplied for $v 2$ in the $l$ th query is latest $(v 2, l-1)$, which by Fact $1(\mathrm{c})$ is same as latest $(v 2, j-1)$.

On the other hand, $V$ value assigned to $v 1$ in $l$ th query is $\operatorname{latest}(v 1, j-1)$. Without loss of generality, assume $v 1$ is the left child. Then, (latest $(v 1, j-$ 1) || latest $(u, j-1) \|$ latest $(v 2, j-1))$ was inserted in List $_{l}$. Moreover, by Claim 2 , and Fact 1(b), these are the only values in $\operatorname{List}_{j-1}$, with middle value latest $(u, j-$ 1 ). Now, suppose for one of these $v$ (i.e. $v 1$ or $v 2$ ), $V(v)$ (i.e. supplied in the $j$ th query) is not the same as latest $(v, j-1)$. But, by the claim that (b) does not hold we have that $(V(v 1) \|$ latest $(u, j-1) \| V(v 2))$ is in $\operatorname{List}_{j-1}$, which leads to a contradiction.

Thus, for $v$ being either child of $u, V(v)$ supplied in the $j$ th query is indeed latest $(v, j-1)$. That completes the nested induction step.

But since, $V(r)$ supplied in the $j$ th query is indeed same as latest $(r, j-1$ ) (as the $\tau$ values are not altered by the adversary), the induction step is proven.

We are now ready to complete the proof of Theorem 1 . We point out again that we use notation from Definition 2.3. Also, recall that we assume, w.l.o.g that all updates returned non- $\perp$ values. Let Verified be the event $\operatorname{Verify}_{x}\left(j, a, \sigma^{\prime}, \tau_{q}\right)=1$.

Let $u$ correspond to the leaf at index $i$ as specified in the final Verify query of A. Then $M_{i}^{q}=$ latest $(u, q)$. This follows from the fact that either the $i$ th leaf was never validly updated, in which case $M_{i}^{q}=M_{i}^{0}=\operatorname{latest}(u, 0)=\operatorname{latest}(u, q)$, or it was updated at $\operatorname{last}(u, q)=l$ to be block $a_{l}$, in which case $M_{i}^{q}=a_{l}=\operatorname{latest}(u, l)$ $=\operatorname{latest}(u, q)$.

Since, data $a$ to be verified at leaf node $u$ corresponding to block number $j$ is different from $M_{j}^{q}$, and hence is different from latest $(u, q)$, Claim 3(a) does not hold. Hence, Claim 3(b) must hold. Let $j$ be the query and $v$ be the non-leaf node in $S_{j}$, such that $(V(\operatorname{left}(v))\|V(v)\| V(\operatorname{right}(v)))$ is not in List $_{j-1}$. Let $M^{\prime}=(V(\operatorname{left}(v))\|V(v)\| V(\operatorname{right}(v)))$, and $\tau^{\prime}=C(v)$, where $C(v)$ is the value supplied by the adversary $\mathrm{A}$ in the $j$ th query. Now $F_{x}\left(M^{\prime}\right)=\tau^{\prime}$, for otherwise PAT would return $\perp$ (see step 1 of INC-MAC-AUX), which is not possible as we 
had assumed w.l.o.g. that no update returns $\perp$. Thus if Verified happens with probability $p$, then in the experiment of Definition 2.1, $F_{x}\left(M^{\prime}\right)=\tau^{\prime}$ happens with probability at least $p$ as well.

\section{Optimizations}

The PAT scheme used a function $F:\{0,1\}^{k} \times\{0,1\}^{\max \{d, m\}+d+1} \rightarrow\{0,1\}^{h}$, and the security of PAT required this function to be a secure MAC. It is well know that if $F$ is a secure pseudorandom function family, then it is also a secure MAC (as in definition 2.1) 7]. The question then boils down to building an efficient PRF from $\max \{d, m\}+d+1$ bits to $l$ bits.

First note that, a simple MAC scheme obtained from a PRF is susceptible to birthday attacks, and hence it is secure only up to at most $2^{h / 2}$ queries. This implies, that $d$ need only be $h / 2$. Thus, one needs to implement a PRF from $3 h / 2$ bits to $h$ bits, with a key of size $k$. In fact, instead of considering a binary tree, one could consider a 4 -ary tree, in which case one needs a PRF from $5 / 2 h$ to $h$. HMAC [9] with SHA-1 is a reasonable option for such a PRF (with $h=160$ ) where the key is fed through the IV of SHA (see [9] for a discussion of keying through the IV).

In such a 4 -ary tree implementation, the number of leaf nodes is $4^{n}$. Thus, the amount of data can be $4^{n} *(h / 2)$, assuming each block of data is 80 bits, i.e. $\mathrm{m}=80$. The number of non-leaf nodes is $\left(4^{n}-1\right) / 3$, and each internal node has memory requirement of $d+h=3 h / 2$ bits. Thus, the memory overhead for auxiliary data is $100 \%$.

In an 8 or $16 \mathrm{~N}$-ary tree implementation, the storage overhead is reduced for the intermediate nodes to a more practical level of less than $43 \%$ or $20 \%$ respectively.

\section{Alternative Schemes}

Definition 6.1 (Universal Hash function family [5]). A set $\mathcal{H}$ of functions with signature $\{0,1\}^{t} \rightarrow\{0,1\}^{n}$ is said to be a Universal Hash Function family if $\operatorname{Pr}_{H}[H(i)=H(j)] \leq 2^{-n}$ for all $i, j$, where the probability is taken over $H$ chosen uniformly at random from $\mathcal{H}$.

Definition 6.2 (XOR-universal function family [8]). A set $\mathcal{H}$ of functions with signature $\{0,1\}^{t} \rightarrow\{0,1\}^{n}$ is said to be an XOR-universal family if $\operatorname{Pr}_{H}[H(i) \oplus$ $H(j)=k] \leq 2^{-n}$ for all $i, j, k$, where the probability is taken over $H$ chosen uniformly at random from $\mathcal{H}$.

In general, if $h 1$ is an XOR-universal hash function family with key $k 1$, from $c$ bits to $c$ bits, then $a+h 1_{k 1}(b)$, is a universal hash function family from $2 c$ bits $(\langle a, b\rangle)$ to $c$ bits.

Instead of describing XOR-MAC [1], we now briefly describe the essential idea behind the PMAC [3] scheme, which is a more advanced version of XOR-MAC.

Let $M$ be a plaintext of length $2^{n}$ blocks, on which PMAC is to be computed. Each block is of length $m$. For each block of $M$ identify a node $u$ with a unique 
address, namely address $(u)$. The data at node $u$ will be called $M(u)$. The PMAC scheme employs a pseudorandom permutation (PRP) $E$ from $m$ bits to $m$ bits with key $x$. The PMAC algorithm also picks a function $H$ randomly and uniformly from a universal hash function family. Thus, the secret key of the PMAC algorithm is $x$ and the function $H$. The PMAC value of $M$ is then just

$$
E_{x}\left(\bigoplus_{i=1}^{2^{n}} E_{x}\left(H\left(M\left(u_{i}\right) \| \operatorname{address}\left(u_{i}\right)\right)\right)\right)
$$

In other words, the function $H$ is used to hash the data value at each node, along with its address, to be the input of the PRP $E_{x}$. The output of all the $E_{x}$ operations is xored and sent through $E_{x}$ once again to obtain the mac value.

Note that this scheme has a critical path of length two ( $E$ operations) in a parallel implementation. Moreover, it allows for fast incremental updates. For example, if the PMAC value of $M$ is $\tau$, then the PMAC value of $M\langle i, a\rangle$ is obtained by first employing $E_{x}^{-1}$ on $\tau$ to get a temporary value $s$. Then, $s$ is updated by xoring it with $E_{x}\left(H\left(M\left(u_{i}\right) \| \operatorname{address}\left(u_{i}\right)\right)\right)$ and $E_{x}\left(H\left(a \| \operatorname{address}\left(u_{i}\right)\right)\right)$, i.e.

$$
s=s \oplus E_{x}\left(H\left(M\left(u_{i}\right) \| \operatorname{address}\left(u_{i}\right)\right)\right) \oplus E_{x}\left(H\left(a \| \operatorname{address}\left(u_{i}\right)\right)\right)
$$

Finally, the new PMAC value is computed as $E_{x}(s)$.

In this section we point out that XOR-MAC can also be extended to be an efficient and parallel IMACAUX scheme. Recall that XOR-MAC was deficient in the sense that it did not allow efficient partial authentication. So to add that feature, we first must use a version of XOR-MAC which uses universal hash functions. The schemes PMAC ([3]) and XECB-MAC ([6]) are such schemes.

We now show how to extend PMAC to allow efficient partial authentication.

This scheme will also have a binary tree structure as the auxiliary data, just as in PAT. The leaves will just be the $2^{n}$ nodes described above in PMAC. As in PAT, each internal node has a $C$ value and a $V$ value. The $V$ value is only computed during updates or verification (i.e. it is secret), and is not stored as a part of the tree (only $C$ values are stored or returned as part of $\sigma$ ). The local MAC is computed using PRP $E$. In particular, the $V$ value at an internal node is computed by an xor-sum of the $V$ values of all its children, and then the $C$ label of this internal node is computed by encrypting the $V$ value with $E$.

The $V$ value at leafs is computed slightly differently. The function $H$ is used to hash the data value at each leaf, along with its address, to be the input of the PRP $E$. The result of this application of $E$ is the $V$ value of the leaf.

We observe that the $V$ value of any internal node $u$ (including the root) is the XOR-sum of the $V$ values of all the leaf nodes under this internal node $u$. By keeping the $C$ labels of the internal node, we assure integrity of a leaf by just checking the path from the leaf to the root. The "address sensitivity" is built into the leaves using the function $H$.

This scheme however has the drawback of having a critical path of a decryption followed by encryption in a parallel implementation. Essentially, all the $C$ values on an update path and the sibling path must first be decrypted, and 
then the $V$ values are updated by the changed value at the leaf, and finally the new $C$ values are obtained by an encryption. Contrast this with INC-MAC-AUX (update) of PAT where all the $F$ operations in step 1 to 3 can be done in parallel.

\section{Systems Issues}

In this section we address several systems issues, which also highlights the motivation behind PAT. Naturally, the comparison of this scheme with Merkle trees and PMAC trees arises.

In any practical tamper proof system, we do not expect to fully parallelize the operations involved in an update or memory authentication. Most likely, the tamper proof system will have a reasonably large cache (i.e. inside the tamper proof device). This would allow the updates to be done in a lazy fashion.

Thus, the PAT tree is never completely updated in the insecure memory after the initialization (unless completely flushed from the cache - an atypical event). When a new data node is brought into cache, all the nodes in the update path between the leaf (i.e. data node) and the lowest ancestor which is already present in the cache, must be brought in (or filled). When a modified (or dirty) node is cast-out (cache cast-out), not only is it updated in the PAT representation in the insecure memory, its parent even if it still remains in cache is assigned a new $\mathrm{V}$ label. The nodes above this parent node are not required to get a new label unless their children are themselves being cast out. Essentially, all nodes which are in the insecure memory, must be consistent with the lowest ancestor which is in cache - as all nodes in the cache are considered authentic.

We point out that entries at higher levels of the tree cover larger areas of memory, eventually covering whole "working sets" and benefiting from "localityof-reference" effects in the caching. The highest levels of the tree will entirely fit in the cache, and so after the initial verify for the first misses, will never be cast-out (and so will never be updated in memory). This will appear as multiple smaller trees with the "root" V's being updated and keep in the internal cache.

Having discussed the benefits of cache, practical high performance solutions will still have to "pipeline" memory and buses. Pipelined memories and buses tend to reach their peak bandwidth when bursting blocks of data. This will imply authentication trees with higher N-ary ( 8 or 16) structures to take advantage of the burst bandwidth. Given the large block size of the nodes, it will be less costly (less hardware buffering in the data path) if a block can be processed in a "pipelined" (higher bandwidth) verification engine as soon as it arrives from the bus.

For PAT this implies pipelining the intermediate level node blocks as a sequence of data bursts from the root to the leaf (top-down), thus having the higher level $\mathrm{V}$ available for the processing of the next lower level. To reduce the hardware buffering in the update (cache cast-out) path, the processing will need to proceed in the same top-down manner phased slightly ahead of the top-down verification (cache fill) so that the previous cache line contents have been saved (update) to external memory before being overwritten by the new contents from the verification path (cache fill). 
In contrast, Merkle Tree updates have a computational dependency from the leaf nodes to the root node (bottom-up) and a top-down dependency only for verifications. This again implies pipelining the intermediate level node blocks as a sequence of data bursts, but this time from the leaf to the root (bottomup) for both verifications and updates. Again, with the bottom-up updates (cache cast-out) phased slightly ahead of the bottom-up verifications (cache fill) to avoid additional hardware buffering. The hash operations for verify can be done in a pipelined or parallel manner, and the updates which were caused by cast-outs at various levels in the tree cache will typically be unrelated and so may also be pipelined. But additional complexity will be involved in the handling of cases where a higher level parent node in the cast-out sequence will require the result from a next lower level child node's update (cast-out) hash computation before being able to begin it's own store and hash operations. This additional delay would then also be reflected in the verification processing to maintain the update to verify or cache block save to fill phase alignment. Observe that this effect is only reduced by hash engines with lower individual block computation latencies; higher bandwidth engines will not reduce the delay for an individual update, only the average delay for multiple parallel updates.

Finally, PMAC Tree updates have a bottom-up computational dependency that is similar to Merkle Trees (leaf to root), but this time only the leaf node to the first intermediate level has the full latency of the crypto computation (hash followed by encryption), the higher levels are simple XORs with very small latency effects. The PMAC tree verification is a comparison between levels and so is fully parallelizable. As in the Merkle Tree case, the pipelining of the intermediate level nodes would be from the leaf to the root (bottom-up) for both verify and update, again with the same phase requirements. Similarly, the verify and the typically unrelated updates may be pipelined. In contrast to Merkle Trees however, the additional complexity will only occur when handling cases where the first intermediate level parent node in the cast-out sequence requires the hash and encrypt result from a data level child's update (cast-out) before being able to begin it's own encrypt and store operation. This additional delay would then need to be reflected in the verify processing to meet the update to verify phase requirement, but in the PMAC case, this will only occur at most once during a cache miss sequence. Unfortunately, the data level processing involved in computing the encrypted hash values is the same for both update (cast-out) and verify (fill), while the processing of the intermediate levels involve encryption for update (cast-out) and decryption for verify (fill). This means that the verify (fill) path will require a crypto engine capable of both the forward and inverse version of the chosen cryptographic algorithm, and this will typically require more gates and cause more potential timing problems.

Acknowledgments. The authors would like to thank several anonymous referees for their helpful comments. 


\section{References}

1. M. Bellare, R. Guerin and P. Rogaway, XOR MACs: New methods for message authentication using finite pseudorandom functions, Advances in Cryptology - Crypto 95 Proceedings, Lecture Notes in Computer Science Vol. 963, D. Coppersmith ed, Springer-Verlag, 1995.

2. M. Bellare, O. Goldreich, S. Goldwasser, "Incremental Cryptography with Applications to Virus Protection", Proc. STOC 1995

3. John Black and Phillip Rogaway, "A Block-Cipher Mode of Operation for Parallelizable Message Authentication", Advances in Cryptology - EUROCRYPT '02, Lecture Notes in Computer Science, Springer-Verlag, 2002

4. M. Blum, W. Evans, P. Gemmell, S. Kannan, M. Naor, "Checking the Correctness of Memories", Algorithmica, Vol 12, pp 223-244, 1994.

5. J. Carter, M. Wegman, "Universal Classes of Hash Functions", JCSS, Vol. 18, 1979, pp 143-154.

6. V.D. Gligor, P.Donescu, "eXtended Electronic Code Book MAC", http:// csrc.nist.gov/encryption/modes/proposedmodes

7. O. Goldreich, S. Goldwasser, and S. Micali, "How to construct random functions", J. ACM, vol. 33, no. 4, 1986.

8. Hugo Krawczyk, "LFSR-based Hashing and Authentication", Proc. Crypto 94, LNCS 839, 1994

9. M. Bellare, R. Canetti, and H. Krawczyk, "Keying hash functions for message authentication", Advances in Cryptology-Crypto '96, 1996.

10. C. S. Jutla, "Encryption Modes with Almost Free Message Integrity", Eurocrypt 2001, LNCS 2045.

11. Moses Liskov, Ronald L. Rivest, David Wagner: Tweakable Block Ciphers, CRYPTO 2002: 31-46

12. M. Luby, "Pseudorandomness and Cryptographic Applications", Princeton Computer Science Notes, Princeton Univ. Press, 1996

13. R. Merkle, "A certified digital signature", Crypto 89, LNCS 435, 1989. 